\title{
An image of the dust sublimation region in the nucleus of NGC 1068^
}

\author{
GRAVITY Collaboration: O. Pfuhl ${ }^{1}$, R. Davies ${ }^{1}$, J. Dexter ${ }^{1}$, H. Netzer ${ }^{8}$, S. Hönig ${ }^{5}$, D. Lutz ${ }^{1}$, M. Schartmann ${ }^{1}$, \\ E. Sturm ${ }^{1}$, A. Amorim ${ }^{19,21}$, W. Brandner ${ }^{22}$, Y. Clénet ${ }^{2}$, P. T. de Zeeuw ${ }^{1,17}$, A. Eckart ${ }^{3,18}$, F. Eisenhauer ${ }^{1}$, \\ N.M. Förster Schreiber ${ }^{1}$, F. Gao ${ }^{1}$, P. J. V. Garcia ${ }^{15,20,21}$, R. Genzel ${ }^{1,4}$, S. Gillessen ${ }^{1}$, D. Gratadour ${ }^{2}$, M. Kishimoto ${ }^{6}$, \\ S. Lacour ${ }^{2,1}$, F. Millour ${ }^{7}$, T. Ott ${ }^{1}$, T. Paumard ${ }^{2}$, K. Perraut ${ }^{12}$, G. Perrin ${ }^{2}$, B. M. Peterson ${ }^{9,10,11}$, P. O. Petrucci ${ }^{12}$, \\ M. A. Prieto ${ }^{23}$, D. Rouan ${ }^{2}$, J. Shangguan ${ }^{1}$, T. Shimizu ${ }^{1}$, A. Sternberg ${ }^{13,14}$, O. Straub ${ }^{1}$, C. Straubmeier ${ }^{3}$, \\ L. J. Tacconi ${ }^{1}$, K. R. W. Tristram ${ }^{15}$, P. Vermot ${ }^{2}$, I. Waisberg ${ }^{1}$, F. Widmann ${ }^{1}$, and J. Woillez ${ }^{16}$ \\ (Affiliations can be found after the references)
}

Received 5 July 2019 / Accepted 1 December 2019

\begin{abstract}
We present near-infrared interferometric data on the Seyfert 2 galaxy NGC 1068, obtained with the GRAVITY instrument on the European Southern Observatory Very Large Telescope Interferometer. The extensive baseline coverage from 5 to $60 M \lambda$ allowed us to reconstruct a continuum image of the nucleus with an unrivaled $0.2 \mathrm{pc}$ resolution in the $K$-band. We find a thin ring-like structure of emission with a radius $r=0.24 \pm 0.03 \mathrm{pc}$, inclination $i=70 \pm 5^{\circ}$, position angle PA $=-50 \pm 4^{\circ}$, and $h / r<0.14$, which we associate with the dust sublimation region. The observed morphology is inconsistent with the expected signatures of a geometrically and optically thick torus. Instead, the infrared emission shows a striking resemblance to the $22 \mathrm{GHz}$ maser disc, which suggests they share a common region of origin. The near-infrared spectral energy distribution indicates a bolometric luminosity of $(0.4-4.7) \times 10^{45} \mathrm{erg} \mathrm{s}^{-1}$, behind a large $A_{K} \approx 5.5\left(A_{V} \approx 90\right)$ screen of extinction that also appears to contribute significantly to obscuring the broad line region.
\end{abstract}

Key words. galaxies: active - galaxies: nuclei - galaxies: Seyfert - techniques: interferometric

\section{Introduction}

NGC 1068 is often regarded as an archetypical Seyfert 2 galaxy (e.g. Bland-Hawthorn et al. 1997). In particular, observations of NGC 1068 played an important role in the early development of unified models of active galactic nuclei (AGN). In this model, opaque material in the equatorial plane obscures the supermassive black hole, accretion disc, and broad emission lines, so that the AGN can only be observed directly from polar directions (Miller \& Antonucci 1983; Antonucci \& Miller 1985). Following the inception of the torus concept (Antonucci 1982, 1984) and the first efforts to explain its physical properties (Krolik \& Begelman 1988), there have been numerous observations of the central structures in this and other AGN from across the electromagnetic spectrum and covering many different tracers. Ideas about the geometry of the central obscurer have evolved and modified significantly over the years. Indeed, in the literature the term "torus" is used to mean a variety of different things. While it was always understood that this structure might be clumpy (Krolik \& Begelman 1988), early calculations adopted a smooth geometry (Pier \& Krolik 1992). The subsequent incorporation of a clumpy structure led to a suite of models that provide very good fits to the near-to-mid infrared spectral energy distribution (SED) of many AGN (Nenkova et al. 2008; Hönig et al. 2006; Stalevski et al. 2012), and which are also consistent with dust reverberation measurements (Koshida et al. 2014). We refer to Netzer (2015) for a detailed review. Models and observations have achieved a level of detail where it is possible to apply a wide range of tests to the

\footnotetext{
* The reconstructed image and interferometric beam are available at the CDS via anonymous ftp to cdsarc.u-strasbg.fr (130.79.128.5) or via http://cdsarc.u-strasbg.fr/viz-bin/ cat/J/A+A/634/A1
}

properties and assumptions used in creating them. Additionally, the data we present here allow a new test to be applied. It concerns the observed morphology of the hot dust close around the AGN. To provide a context for this, we highlight a few key observations pertinent to understanding the central few parsecs around the black hole in NGC 1068 as follows.

The detection of broad permitted emission lines in polarised light (Antonucci \& Miller 1985) represents a cornerstone of the AGN unification scheme (Antonucci 1993), which postulates that the observed properties of an AGN mainly depend on inclination. Cameron et al. (1993) pointed out that other structures must also be present on small scales in NGC 1068 since the midinfrared (MIR) continuum is extended on scales of $\sim 1^{\prime \prime}$ rather than dominated by a central source. Using higher resolution data, Bock et al. (2000) showed there was a central $<0.2^{\prime \prime}$ core, which they associated with the torus, and a tongue extending $\sim 1^{\prime \prime}$ along a north-south axis that mimics the inner part of the radio jet (Gallimore et al. 1996a), which is thought to be launched in a northerly direction and bends to the north-east when it encounters a dense molecular cloud.

Early $K$-band speckle and interferometry work found hot dust emission coming from multiple components on scales of 400 mas to a few mas (Weigelt et al. 2004; Wittkowski et al. 2004). Observations with MIDI at $10 \mu \mathrm{m}$ indicated the presence of multiple dust components with a central $600-800 \mathrm{~K}$ elongated dust core. The first component is a $800 \mathrm{~K}$, disc-like structure about $1.4 \times 0.5 \mathrm{pc}$ in size at a position angle (PA) of approximately $-45^{\circ}$ (Jaffe et al. 2004; Raban et al. 2009; López-Gonzaga et al. 2014). A second, cooler $\sim 300 \mathrm{~K}$ component, $3 \times 4 \mathrm{pc}$ in size, was identified with the "body" of the torus (Raban et al. 2009).

The presence of a complex structure consisting of multiple components is supported by X-ray spectra, indicating a compact 
high column density $N_{\mathrm{H}}=10^{25} \mathrm{~cm}^{-2} \mathrm{X}$-ray reflector with lower column $N_{\mathrm{H}}=10^{23} \mathrm{~cm}^{-2}$ clouds on more extended scales (Bauer et al. 2015). This does not necessarily imply anything about a dusty structure, since the X-ray absorption could be through dust-free clouds in the optically hidden broad line region (BLR). The lack of $\operatorname{Br} \alpha$ detection led Lutz et al. (2000) to put a lower limit on the extinction corresponding to $A_{V} \sim 100 \mathrm{mag}$, or an equivalent column of $n_{\mathrm{H}} \sim 10^{23} \mathrm{~cm}^{-2}$ for a standard dust-to-gas ratio.

A maser disc is seen extending up to $\sim 15$ mas ( $1 \mathrm{pc}$ ) from the AGN, with velocities reaching $\pm 330 \mathrm{~km} \mathrm{~s}^{-1}$ (Gallimore et al. 1996b; Greenhill \& Gwinn 1997; Gallimore et al. 2004). In addition, Greenhill \& Gwinn (1997) noted that the $\sim-45^{\circ}$ position angle of the disc rotation axis differs from that of the jet by 30$40^{\circ}$. As such, there is clearly some warping on sub-pc scales in NGC 1068, and any description of the geometry needs to be associated with a particular structure or radial scale. The maser disc can be described by a thin edge-on $\left(i \sim 80^{\circ}\right)$ disc. Studies based on SED modelling also favour torus inclinations between $70^{\circ}$ (Hönig et al. 2007; Lopez-Rodriguez et al. 2018) and $90^{\circ}$ (Hönig et al. 2008). Attempts to model the three dimensional orientation of the system found that the northern narrow line region (NLR) cone is directed towards the observer and the southern part away from the observer (Packham et al. 1997; Kishimoto 1999).

Near-infrared (NIR) integral field adaptive optics observations revealed streamers in the $\mathrm{H}_{2} 1-0 \mathrm{~S}(1)$ line, tracing hot molecular gas, which were interpreted as infalling material on a few tens of parsecs scales (Müller Sánchez et al. 2009). They also provided the first glimpse of a molecular structure on a $\sim 10$ pc scale.

Gratadour et al. (2015) obtained polarimetric imaging with SPHERE. This showed a bicone structure perpendicular to the maser disc, probably related to outflows, as well as an elongated patch aligned with the central molecular structure but extending further out $(R \approx 25 \mathrm{pc})$, which they interpreted as the less dense outskirt of the putative torus.

ALMA observations of the $432 \mu \mathrm{m}$ continuum and $\mathrm{CO}(6-$ 5) molecular line resolved a $7-10 \mathrm{pc}$ thick-disc like structure (Gallimore et al. 2016; García-Burillo et al. 2016). As expected for a Seyfert 2 galaxy, observational data argue for a system close to edge-on. Based on the linewidth of the spatially resolved $\mathrm{HCN}$ and $\mathrm{HCO}^{+}$lines in more recent observations of this structure, Imanishi et al. (2018) argue for turbulent motions with $\sigma=70$ $80 \mathrm{~km} \mathrm{~s}^{-1}$, of similar order as the rotational velocity. Impellizzeri et al. (2019) find similar line widths close to the nucleus. GarcíaBurillo et al. (in prep.) detected the molecular disc in $\mathrm{CO}(2-1)$ and (3-2) at a PA $\sim 115^{\circ}$ with an extension of $30 \mathrm{pc}$.

Taken together, these observations, while still being consistent with and requiring the existence of a nuclear obscuring structure, are incompatible with geometrically thick clumpy torus models that attempt to account for all of the nuclear nearto-mid infrared continuum. Vollmer et al. (2018) propose a resolution to this discrepancy by constructing a model which has a thin disc on scales $\lesssim 1 \mathrm{pc}$ and a thick disc on scales of $1-10 \mathrm{pc}$, the structure of which is determined by inflow from larger scales in the host galaxy. In their model, a magnetocentrifugal wind is launched at the boundary between the discs, and accounts for the elongated polar structures seen in many MIR interferometric measurements (López-Gonzaga et al. 2016). This multi-component model was able to account for a wide variety of detailed observations when applied to NGC 1068. A similar approach has been described by Hönig (2019) who posits a dusty disc with small to moderate scale height, through which
Table 1. Basic parameters of NGC 1068 taken from the literature.

\begin{tabular}{lll}
\hline \hline Parameter & Value & Source \\
\hline Distance & $14.4 \mathrm{Mpc}$ & {$[1]$} \\
$M_{\mathrm{BH}}$ & $0.8-1.7 \times 10^{7} M_{\odot}$ & [2], [6], [7], [8] \\
$L_{\text {bol }}$ & $0.4-4.7 \times 10^{45} \mathrm{erg} \mathrm{s}^{-1}$ & Sect. 2.1, [3], [4], [5] \\
\hline
\end{tabular}

References. [1]: Bland-Hawthorn et al. (1997); [2]: Lodato \& Bertin (2003); [3]: Marconi et al. (2004); [4]: Bauer et al. (2015); [5]: LopezRodriguez et al. (2018); [6]: Greenhill et al. (1996); [7]: Huré (2002); [8]: Gallimore \& Impellizzeri (2019).

gas flows inwards and is then unbound in a dusty wind launched at the inner puffed-up rim by radiation pressure from the AGN.

\section{Prerequisites and observations}

\subsection{Bolometric luminosity and sublimation distance}

For consistency with most of the NGC 1068 literature, we adopt the "standard" distance of $14.4 \mathrm{Mpc}$ (Bland-Hawthorn et al. 1997 ) but note that $d \approx 16.5 \mathrm{Mpc}$ would arise from Virgocentric infall velocity $1117 \mathrm{~km} \mathrm{~s}^{-1}$ (Mould et al. 2000, NED ${ }^{1}$ ) and the Hubble constant $\left(H_{0}=67.8 \pm 0.9 \mathrm{~km} \mathrm{~s}^{-1} \mathrm{Mpc}^{-1}\right)$ of Planck Collaboration XIII (2016).

The estimated black hole mass of up to $M_{\mathrm{BH}} \approx 1.7 \times 10^{7} M_{\odot}$ (see Table 1) corresponds, for a solar metallicity gas, to an Eddington luminosity of $L_{\text {Edd }} \approx 2.5 \times 10^{45} \mathrm{erg} \mathrm{s}^{-1}$.

The literature reports bolometric luminosities between $L_{\mathrm{bol}} \approx$ $0.5 \times 10^{45} \mathrm{erg} \mathrm{s}^{-1}$ (Lopez-Rodriguez et al. 2018) based on SED modelling and $L_{\mathrm{bol}} \approx 1.6 \times 10^{45} \mathrm{erg} \mathrm{s}^{-1}$ based on MIR data (Raban et al. 2009). Prieto et al. (2010) on the other hand found a significantly lower luminosity of $\sim 9 \times 10^{43} \mathrm{erg} \mathrm{s}^{-1}$.

Our estimates of $L_{\text {bol }}$ are based on various X-ray and optical observations of the source. When we compared typical luminosity probes, we found that the X-ray based $L_{\text {bol }}$ estimates are significantly lower than the estimates from ionised lines. Based on detailed modelling, Bauer et al. (2015) infer an X-ray luminosity of $L_{2-10 \mathrm{keV}} \approx 2 \times 10^{43} \mathrm{erg} \mathrm{s}^{-1}$, which includes a number of X-ray components and a detailed SED fitting of the source. Using broadband X-ray data Ricci et al. (2017) find an intrinsic $L_{14-195 \mathrm{keV}} \sim 5 \times 10^{42} \mathrm{erg} \mathrm{s}^{-1}$. Moreover, recent observations of Marinucci et al. (2016) find X-ray variability, due to column density variations. The authors infer an intrinsic $L_{2-10 \mathrm{keV}} \approx$ $7 \times 10^{43} \mathrm{erg} \mathrm{s}^{-1}$. The X-ray estimates combined with the Marconi et al. (2004) bolometric correction factor, give $L_{\text {bol }}=0.4-1.4 \times 10^{45} \mathrm{erg} \mathrm{s}^{-1}$. An estimate based on the nuclear luminosity of the $12 \mu \mathrm{m}$ continuum can be made using the relations of Asmus et al. (2015), together with additional relations from Winter et al. (2012). These yield $L_{\text {bol }} \sim 1.8 \times 10^{45} \mathrm{erg} \mathrm{s}^{-1}$. For the optical data we use estimates based on two combinations of three narrow emission lines: $\mathrm{H} \beta \lambda 4861$, [OIII] $\lambda$ 25007, [OI] $\lambda 6300$. The expressions are taken from Netzer (2009) and make use of reddening corrected line fluxes and galactic type extinction. The line fluxes are taken from Storchi-Bergmann et al. (1995). The resulting bolometric luminosities are $2.3 \times 10^{45} \mathrm{erg} \mathrm{s}^{-1}$ based on the [OIII] and [OI] lines, and $4.7 \times 10^{45} \mathrm{erg} \mathrm{s}^{-1}$ based on the $\mathrm{H} \beta$ and [OIII] lines. An early estimate, using scattered light from an off-nuclear cloud that arguably sees the hidden nucleus, also

1 The NASA/IPAC Extragalactic Database (NED) is operated by the Jet Propulsion Laboratory, California Institute of Technology, under contract with the National Aeronautics and Space Administration. 
favoured luminosities rather in excess of $10^{45} \mathrm{erg} \mathrm{s}^{-1}$ (Miller et al. 1991). The large range of luminosity estimates $L_{\mathrm{bol}}=0.4-$ $4.7 \times 10^{45} \mathrm{erg} \mathrm{s}^{-1}$ reflects the extreme extinction of the source. In any case, NGC 1068 is radiating close to the Eddington limit.

Theoretical and observational work argue for a strong correlation between the NIR size and the AGN luminosity (Suganuma et al. 2006; Kishimoto et al. 2011; Koshida et al. 2014; GRAVITY Collaboration 2019). In the standard picture, the NIR emission originates from the dust sublimation region, which is determined by the dust sublimation temperature $T_{\text {sub }}$. The temperature at which dust sublimates depends on the grain size and composition and ranges from $T_{\text {sub }} \approx 1500 \mathrm{~K}$ for silicate (Si) grains to $T_{\mathrm{sub}} \approx 2000 \mathrm{~K}$ for graphite $(\mathrm{C})$ grains (Baskin \& Laor 2018). By assuming a grain size distribution typical for the interstellar medium (ISM), the mean sublimation distance can be calculated for graphite and silicate dust as $R \approx a_{\mathrm{X}} \times L_{46}^{1 / 2}[\mathrm{pc}]$ with $a_{\mathrm{C}}=0.5$ and $a_{\mathrm{Si}}=1.3$ (e.g. Barvainis 1987; Netzer 2015; for grain size dependence see Baskin \& Laor 2018). However, there is evidence both from SED fitting (Mor \& Netzer 2012) and from dust reverberation mapping (Kishimoto et al. 2007; Koshida et al. 2014) that, close to the AGN, one may find only larger graphite grains. Since, at the same distance from the AGN, large grains are cooler than small grains, one might naturally expect to find a distribution of large graphite grains at the smallest radii. In addition changes in the brightness of the AGN may lead to changes in the dust sublimation radius (Kishimoto et al. 2013) or instead, depending on the dust distribution, its temperature (Schnülle et al. 2015). As such, the sublimation radius is really rather a sublimation region according to grain size and species (e.g. Hönig \& Kishimoto 2017; Baskin \& Laor 2018). Empirical studies have found $R_{2.2 \mu \mathrm{m}} \approx 0.4 \mathrm{pc} \times\left(L_{\mathrm{AGN}} / 10^{46} \mathrm{erg} \mathrm{s}^{-1}\right)^{1 / 2}$ (assuming the $V$ band to $R_{\text {sub }}$ relation from Koshida et al. 2014, and using the conversion $L_{\mathrm{AGN}} \sim 8 L_{5500 \AA}$ from Netzer 2015), which corresponds to a good approximation to the sublimation radius of graphite. For NGC 1068 the observed relation from Koshida et al. (2014) predicts a dust sublimation radius in the range $R_{2.2 \mu \mathrm{m}} \approx 0.08-0.27 \mathrm{pc}$, for the luminosity range quoted in Table 1.

\subsection{Measured column densities}

Numerous studies have tried to estimate the column density obscuring the line-of-sight to the central region of NGC 1068. Early CO/HCN measurements of the central few arcsec suggested column densities of $N_{\mathrm{H}} \sim 0.2-1 \times 10^{23} \mathrm{~cm}^{-2}$ (Planesas et al. 1991; Tacconi et al. 1994; Sternberg et al. 1994). Because the beam is relatively large, these measurements may be affected by the complex structures in the nuclear region, for example the bright $\mathrm{CO}$ emission about $1^{\prime \prime}$ west of the nucleus. More recent higher resolution observations focus on the central molecular structure. Imanishi et al. (2018) found gas masses of $2 \times 10^{6} M_{\odot}$ within $14 \mathrm{pc}$ based on ALMA observations of HCN 3-2 and HCO tracers, which imply a column density of $N_{\mathrm{H}} \sim 2 \times 10^{23} \mathrm{~cm}^{-2}$. ALMA $432 \mu \mathrm{m}$ continuum observations by García-Burillo et al. (2016) inferred gas masses of $M_{\text {gas }}=$ $10^{5} M_{\odot}$ within a structure of $7 \mathrm{pc}$ diameter, suggesting a column density of $8 \times 10^{22} \mathrm{~cm}^{-2}$.

In the infrared, ISO upper limits on the Brackett $\alpha$ broadline emission led Lutz et al. (2000) to place a lower limit on the extinction $A_{\mathrm{Br} \alpha, 4.05} \geq 2.4$, which corresponds to $A_{K} \geq 6$. For an ISM-like gas-to-dust ratio, the optical extinction can be converted into a column density. Based on X-ray calibrations, the relationship between the hydrogen column density $N_{\mathrm{H}}$ and the optical extinction $A_{V}$ is $N_{\mathrm{H}}\left(\mathrm{cm}^{-2}\right)=a \times 10^{21} A_{V}(\mathrm{mag})$, where $a$ has a value ranging from about 1.79-2.21 (Predehl \& Schmitt 1995; Güver \& Özel 2009). Therefore, the observed extinction implies a hydrogen column density of $N_{\mathrm{H}} \gtrsim(1.8 \pm 0.6) \times$ $10^{23} \mathrm{~cm}^{-2}$.

The gas masses found by Müller Sánchez et al. (2009) imply a column density of $N_{\mathrm{H}} \sim 3 \times 10^{24} \mathrm{~cm}^{-2}$ assuming a source size of $0.2^{\prime \prime}$ and a hot-to-cold gas mass ratio of $M_{\mathrm{H}_{2}}^{1-0 S(1)} / M_{\mathrm{H}_{2}}^{\text {total }} \sim 10^{-6}$. Lopez-Rodriguez et al. (2018) estimated $N_{\mathrm{H}}=5 \times 10^{23} \mathrm{~cm}^{-2}$ based on $2-400 \mu \mathrm{m}$ clumpy torus SED fitting over a $0.4^{\prime \prime}$ aperture.

Generally larger column densities are found in the X-rays, which could partially originate from dust-free gas. Various studies found consistently a column density of $N_{\mathrm{H}} \sim 1 \times 10^{25} \mathrm{~cm}^{-2}$ (with a $50 \%$ coverage) based on $3-100 \mathrm{keV}$ data (Matt et al. 1997; Bauer et al. 2015) as well as based on broad-band 0.3$150 \mathrm{keV}$ X-ray model fitting (Ricci et al. 2017).

Overall the different measurements argue for a mean column density of $N_{\mathrm{H}} \sim 10^{23}-10^{24} \mathrm{~cm}^{-2}$ within the central few parsecs to tens of parsecs. The higher column density towards the X-ray emitting corona in the very centre may largely originate in dustfree gas (Risaliti et al. 2002; Netzer 2015; Davies et al. 2015).

\subsection{Extinction correction}

Throughout the paper we have assumed the same extinction law as can be found in the highly absorbed Galactic Centre region (Fritz et al. 2011). For wavelength $<2.6 \mu \mathrm{m}$ we assumed an extinction slope of $A(\lambda) \propto \lambda^{-2.05}$. At larger wavelengths the extinction shows spectral features, which we interpolated based on the extinction published by Fritz et al. (2011). Some useful conversions are: $A_{V} / A_{\mathrm{Br} \gamma} \approx 20, A_{\mathrm{Br} \gamma} / A_{\mathrm{Br} \alpha}=2.5$, $A_{\mathrm{Br} \gamma} / A_{8.76 \mu \mathrm{m}}=1.22$ and $A_{\mathrm{Br} \gamma} / A_{12.4 \mu \mathrm{m}}=1.86$.

\subsection{Interferometric observations}

The observations were carried out with the GRAVITY instrument, which interferometrically combines either four $8 \mathrm{~m}$ Unit Telescopes (UTs), or four $1.8 \mathrm{~m}$ Auxiliary Telescopes (ATs), of the European Southern Observatory (ESO) Very Large Telescope (VLT). Observations of NGC 1068 included both of these, and provided 3 milli-arcsec (mas) resolution $K$-band $(2.2 \mu \mathrm{m})$ continuum imaging. For a detailed description of the instrument and the data analysis we refer to GRAVITY Collaboration (2017). In brief, the light of the four telescopes is extracted into monomode fibres (Pfuhl et al. 2012, 2014) for two positions on the sky and then interfered in the beam combiner for all six baselines of the interferometer. The fibre diameters of 56 mas and 250 mas are matched to the $2.2 \mu \mathrm{m}$ diffraction limited beams of an $8 \mathrm{~m}$ UT and $1.8 \mathrm{~m}$ AT respectively. In a "normal" singlefield observation, the light of the science target is split 50/50 and fed into two channels, the fringe-tracker and the science spectrometer. The fringe-tracker is used to correct the fast atmospheric optical path variations with typical integration times of 1-10 milli-seconds (Lacour et al. 2019). This allows coherent integration times with the science spectrometer up to $\sim 100 \mathrm{~s}$. The interferometric observations of NGC 1068 are challenging due to the complex nature of the object. The central $1^{\prime \prime}$ shows extended continuum as well as line emission. The bright core has been partially resolved with speckle data obtained at the 6$\mathrm{m}$ telescope of the Special Astrophysical Observatory in Russia (Weigelt et al. 2004) as well as with adaptive optics assisted 8-m telescope data from the VLT (e.g. Rouan et al. 2019). Overall there is a significant contribution of coherent flux on all spatial scales, which can be probed with the VLT interferometer (VLTI). 
The visibilities range from $44 \%$ on the shortest AT baselines to $\sim 0.1 \%$ on the longest UT baselines. In order to reach sufficient signal-to-noise, we decided to feed all the light to the fringetracker channel. This provided us with a factor two more signal on the fringe-tracker at the expense of spectral resolving power (The fringe-tracker has five spectral channels across the $K$-band, corresponding to $R \sim 20$ ).

The observations ${ }^{2}$ were taken on 20 November 2018 (1:00-5:20 UTC) with the UT array and on 23 December 2018 (1:30-3:30 UTC) with the AT compact array. The UT observations on November 20th had excellent conditions with seeing measured by the differential image motion monitor (DIMM) between $0.47-0.65^{\prime \prime}$ and 7-13 ms (optical) coherence time. The conditions during the AT observations were almost as good with the seeing ranging from 0.43 to $0.75^{\prime \prime}$ and a coherence time between 4 and $8 \mathrm{~ms}$. In total we obtained $45 \mathrm{~min}$ with the UTs and $30 \mathrm{~min}$ with the ATs of integration time on source. The science observations were bracketed by observations of close calibrator stars HIP $54\left(m_{K}=7.95\right)$, HIP16739 $\left(m_{K}=8.93\right)$ and HIP $17272\left(m_{K}=8.34\right)$. We used the standard pipeline (Lapeyrere et al. 2014; GRAVITY Collaboration 2017) to reduce and calibrate the data.

\subsection{Adaptive optics based photometry}

The combination of four telescopes allows us to simultaneously obtain the coherent as well as the incoherent (absolute) flux of the object. At the same time, the acquisition camera (Anugu et al. 2018) provides an estimate of the Strehl ratio (in $H$-band). By comparing the observed flux of NGC 1068 with observations of known calibrator stars HIP $17272\left(m_{K}=8.34\right)$, HIP 16739 $\left(m_{K}=8.93\right)$ and HIP $54\left(m_{K}=7.95\right)$ we derived an effective magnitude of NGC 1068. As previously described, NGC 1068 is resolved even on scales of a single telescope. This means that the fibre-injected flux depends on the size of the fibre mode on sky. With the UTs, we find a total flux of $310 \pm 80 \mathrm{mJy}$ within the fibre beam $\left(D_{\text {fibre }} \approx 56\right.$ mas). This corresponds to a magnitude of $m_{K}=8.33 \pm 0.25$. Overall, our flux measurement is in good agreement with the previously reported flux of the central component $350 \mathrm{mJy}$ by Weigelt et al. (2004) and with Rouan et al. (2019), who found a central $m_{K}=8.65$.

Weigelt et al. (2004) used speckle data to derive the flux distribution of the central few 100 mas. They modelled the data with two elongated Gaussian components, a central component with a full width at half maximum (FWHM) of 18/39 mas contributing $F_{1}=350 \mathrm{mJy}$ at $\mathrm{PA} \approx-20^{\circ}$, and a larger structure with a FWHM of 400 mas that had a quite uncertain flux estimate of $F_{2}=30-230 \mathrm{mJy}$. The first component has a FWHM significantly smaller than the fibre mode (56/250 mas). Therefore the component is fully injected into our GRAVITY fibres, which agrees with our flux measurement. The 400 mas component however is only partially injected. Less than $30 \%$ (9-69 mJy) of the large component is injected into the fibres using ATs and only $\sim 2 \%(<5 \mathrm{mJy})$ is injected into the fibres using the UTs.

\section{Interferometric data and image reconstruction}

The interferometric data reveal significant structures on all spatial scales. In Fig. 1 we show the calibrated visibilities from the AT and UT observations. For comparison we show the early

2 ESO Telescopes at the La Silla Paranal Observatory programme IDs 0102.B-0667, 0102.C-0205 and 0102.C-0211. speckle data from Weigelt et al. (2004) obtained with a single telescope. The observed visibilities cannot be approximated with a simple symmetric model like a Gaussian or a uniform disc. Also, as shown in the figure, a ring model fails to reproduce the data. This is however not surprising, given the large asymmetries in the source intensity distribution indicated by the highly non-zero closure phases (see Fig. 2). The complexity of the visibility and closure phase data prevent us from using simple model fitting to interpret the observations. Encouraged by the excellent quality of the data and the large uv coverage, we therefore turned to standard image reconstruction codes to analyse the data.

\subsection{An image of the dust sublimation region}

We used the publicly available MiRA tool (Thiébaut 2008) to reconstruct an image based on the visibility and closure phase data. A "grey image" was reconstructed assuming that the intensity distribution on sky $I(\alpha, \beta)$ is independent of the spectral channel across the observed $K$-band. We normalised the visibility by a factor 0.5 to match the visibilities at the smallest spatial frequencies. This means that we reconstruct $50 \%$ of the photometric flux in our image $(\approx 155 \mathrm{mJy})$. The remaining flux originates at larger scales and/or is smoothly distributed. Figure 3 shows the reconstructed image using MiRA with a 1211 smoothness prior (threshold $5 \times 10^{-4}$ ) on a $200 \times 200$ pixel grid with a spatial scale of $0.4 \mathrm{mas} /$ pixel. As an initial guess, we used a single Gaussian image with 5 mas FWHM. The final reconstructed image was then convolved with a Gaussian beam of $F W H M 3.1 \times 1.1$ mas and $\mathrm{PA}=48.6^{\circ}$, which corresponds to 0.8 times the (UT array) interferometric beam. In order to asses the systematic uncertainty of the image, we repeated the reconstruction using different penalty functions (for more information, refer to Thiébaut 2008; Thiébaut \& Young 2017) such as a smoothness prior, (i.e. a penalty on sharp transitions in the image), a compact prior (i.e. penalty on large radii) and a l2l1 smoothness prior (i.e. a prior able to maintain sharp transitions in the image). We stepwise optimised the gain and hyper parameters, where applicable. The resulting images varied but the central structure hardly changed in shape and amplitude. In a second step, we determined the reliability of individual detections in the image by repeating the reconstruction with temporal and bandwidth restricted subsets of the data (with and without the bluest $1.995 \mu \mathrm{m}$ channel which is partially contaminated by metrology laser light). Spurious sources, which were not present throughout the repeated imaging attempts likely correspond to the reconstruction of noise in the data. This noise floor has a rootmean-square $(\mathrm{rms})$ of $\sim 7 \mathrm{mJy}^{\text {beam }^{-1}}$. In addition, the image reconstruction methods used here, where calibrated phases are not available, assume positive fluxes. Therefore, a reconstructed image never contains negative values, unlike single-dish dark- or sky-subtracted images. Numerous spurious noise sources can be seen in Fig 3. The central ring-like structure is highly significant at $5-7 \sigma$. The surrounding features to the north-east and north-

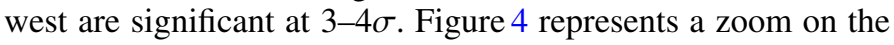
significant central structure.

The image shown in Fig. 4 features an extended structure with a central hole, which can be described as a 3/4 ring with a radius of $r \approx 0.24 \pm 0.03 \mathrm{pc}(3.5 \pm 0.3$ mas $)$, position angle $\mathrm{PA}=-50 \pm 4^{\circ}$ and an inclination of $i=70 \pm 5^{\circ}$. These parameters are summarised in Table 2. The extent of the NIR structure resembles in size and orientation the observed $10 \mu \mathrm{m}$ emission, yet at much higher resolution. The size of the inner bright ring-like structure matches remarkably well the expected dust sublimation distance of graphite grains in the radiation field 


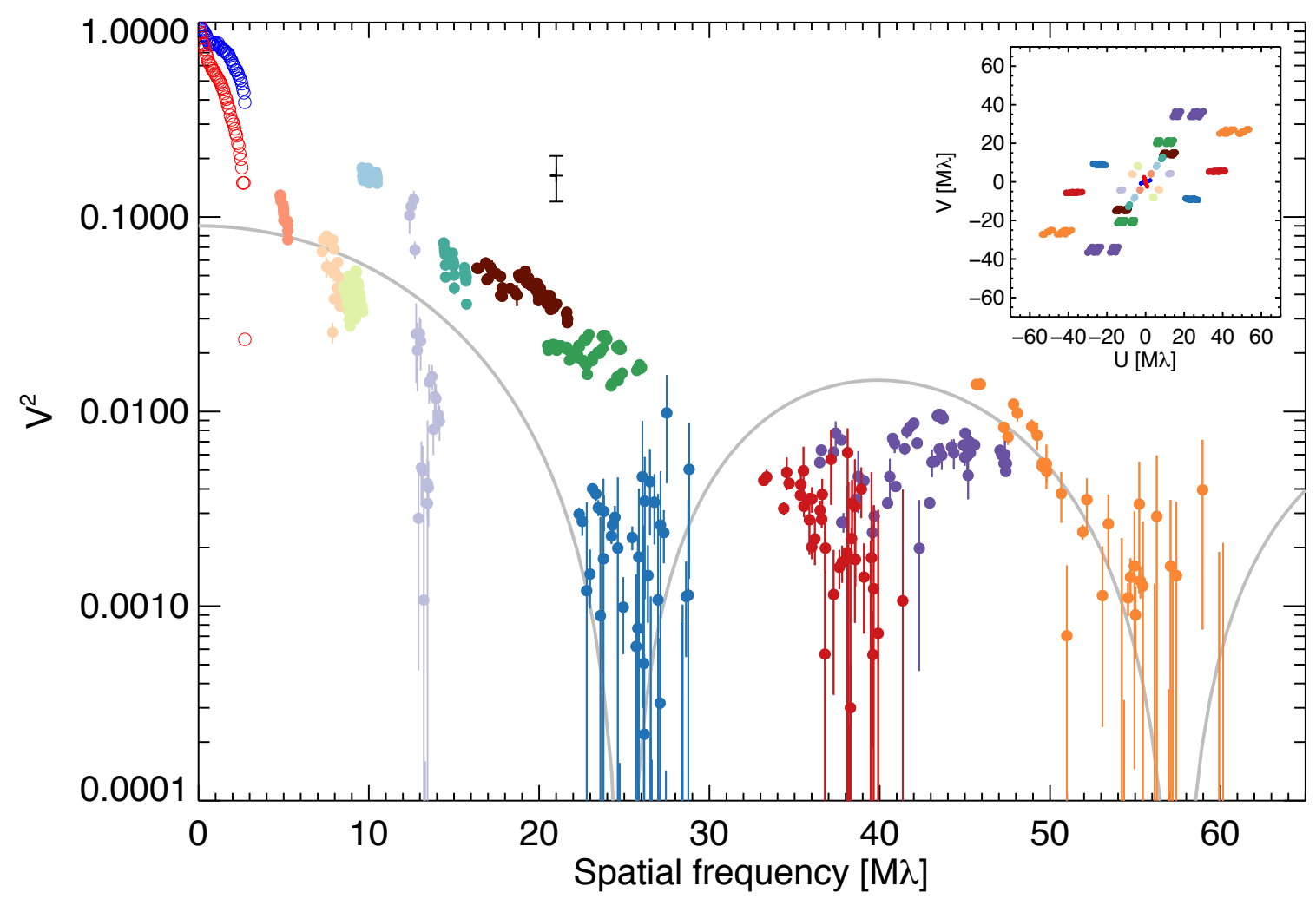

Fig. 1. Squared visibilities measured for NGC 1068. The light coloured filled data points were obtained with the AT array in compact configuration (spatial frequencies $5<f<17 M \lambda$.). The high spatial frequency data were taken with the UT array. The small inset shows the corresponding UV coordinates. The open blue and red symbols (spatial frequencies $f<4 M \lambda$ ) represent speckle data from Weigelt et al. (2004), which are shown only for comparison; they were not used for the image reconstruction. The data point and error bar at a spatial frequency of $21 M \lambda$, lying above our new data, shows the only previous NIR VLTI detection of NGC 1068 (Wittkowski et al. 2004). For comparison, the grey continuous line shows the visibility of a thin ring-shaped emission region with a radius of 3.3 mas and a width of 0.5 mas scaled to a maximum visibility of 0.3 . While this reflects the general shape of the visibility distribution, it is a poor fit and indicates that more complex structures are present.

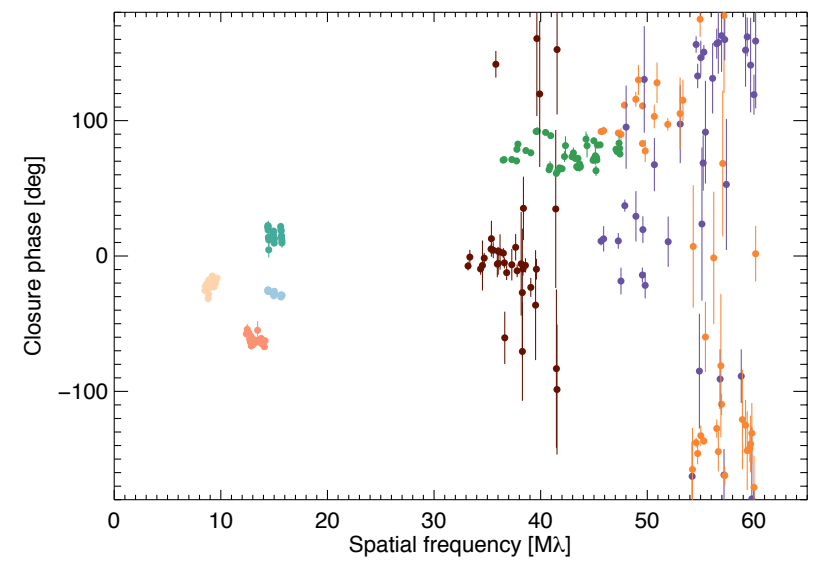

Fig. 2. Closure phase data of NGC 1068. See Fig. 1 for the explanations of the symbols. The highly non-zero closure phases indicate strong source asymmetries on all spatial scales.

of a central engine. The south-western ridge is about a factor two brighter than the north-eastern side of the ring-structure. The north-east orientation of the jet and the ionised outflow, which are approaching the observer (Gallimore et al. 2004), suggests that the south-western ridge corresponds to the nearside of the emission ring (but see Sect. 4.4 for an alternative interpretation). The three dimensional orientation is reinforced if the radio continuum emission (Gallimore et al. 2004) is aligned close to the NIR emission. We find a striking resemblance between the maser emission locations and the bright south western rim of the $K$-band image. Since maser emission is beamed towards the observer, the emitting clouds must lie between the source (AGN) and the observer, and hence the maser emission should originate from the near-side. This alignment, discussed in Sect. 3.3, supports the association of the ring-like structure with the dust sublimation region around the AGN, and it means that the near-side of the dusty ring is not only observable but also brighter than the far-side. Those findings are very difficult to reconcile with geometrically thick clumpy torus models, which can only reproduce a NIR ring-like structure in systems that are relatively face-on, and struggle to make the near side of the ring brighter than the far side (the near and far sides are given by other observations, notably Kishimoto 1999 and Gallimore et al. 2016, as indicated in Sect. 1).

\subsection{Disc height}

The observed width of the ring structure is consistent with it being unresolved. We can place a firm upper limit on the width of the ring of $<0.5$ mas or $0.035 \mathrm{pc}$. If this is interpreted in terms of a vertical thickness, then the scale height of the ring must be $h / r<0.14$.

\subsection{Cospatial dust and maser disc}

Maser emission requires a high velocity coherence of the maser emitting gas as well as a population inversion of the masering 


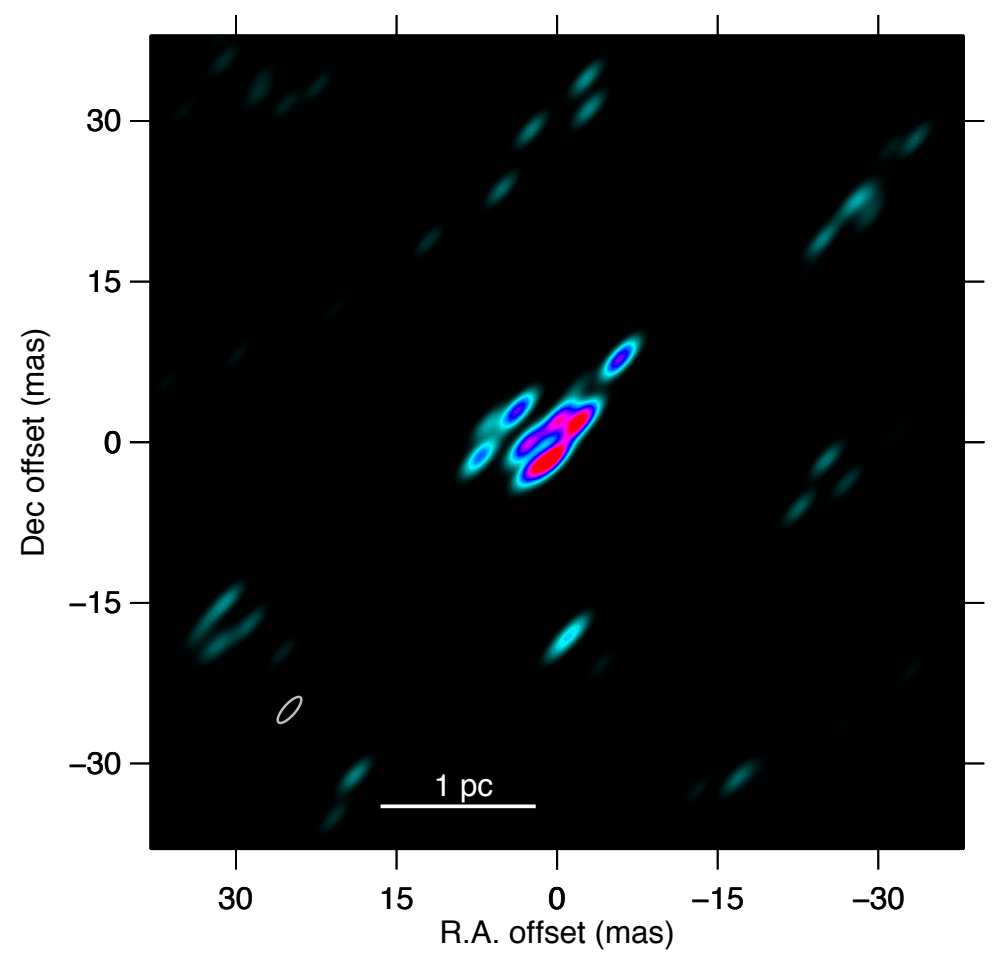

$\left(\mathrm{mJy}_{\text {beam }}{ }^{-1}\right)$

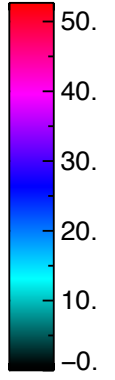

Fig. 3. Reconstructed image of the central $5.1 \times 5.1 \mathrm{pc}$ of NGC 1068 . The elliptical beam, which was used to convolve the image, is shown in the lower left. Details of the reconstruction, which only allows positive sources, are given in Sect. 3.1. The central structures are robust to changing the reconstruction parameters. The fainter features towards the edge of the field, with fluxes close to the noise floor of $\sim 7 \mathrm{mJy}^{\text {beam }}{ }^{-1}$ rms (i.e. dark cyan in this colour scale), are uncertain.

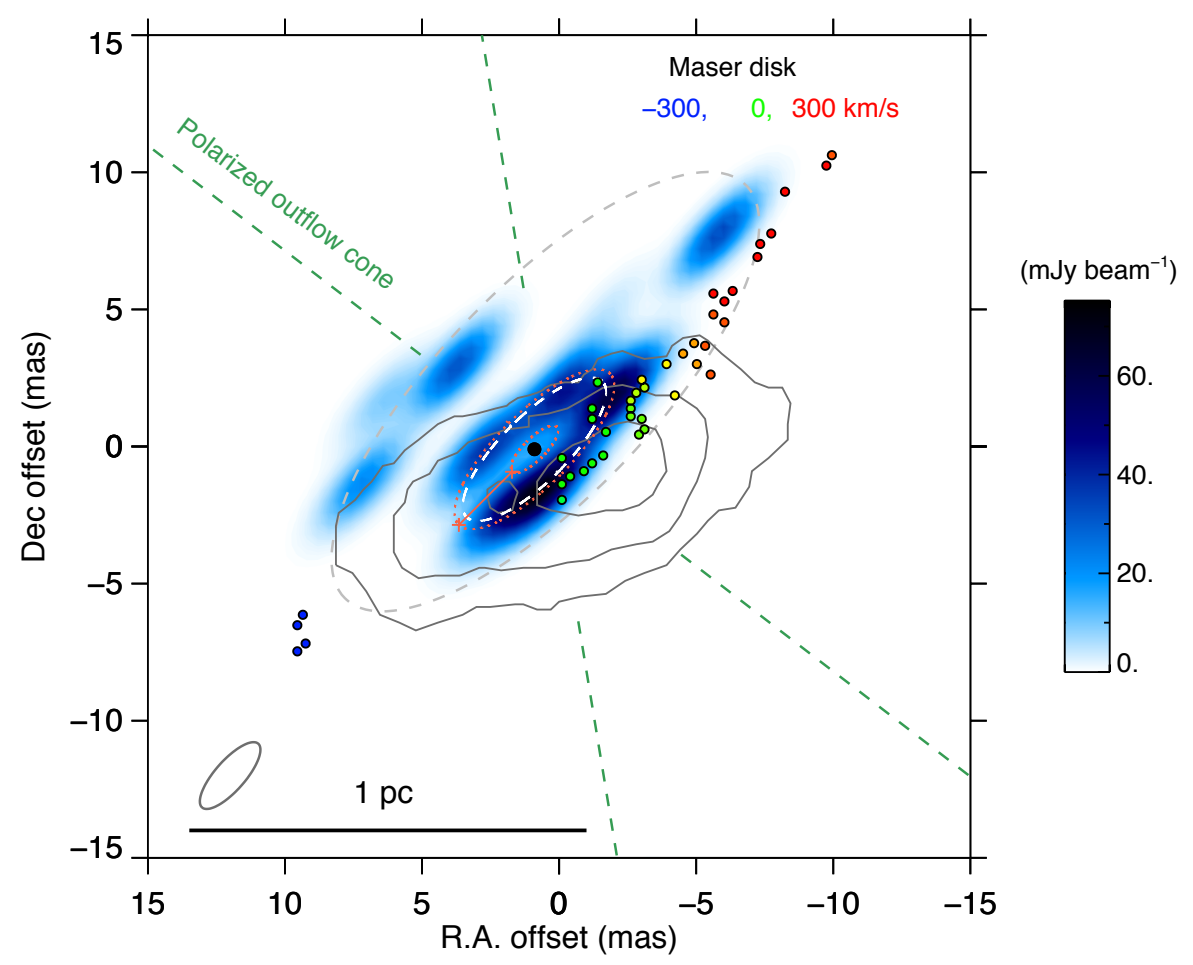

Fig. 4. Reconstructed image of the inner $2.1 \times 2.1 \mathrm{pc}$ of NGC 1068 (i.e. the central region of Fig. 3). The best fitting circular ring is shown in dashed white $(r=0.24 \mathrm{pc}$ (3.5 mas). The dust sublimation sizes for the range of bolometric luminosities $(\sim 0.08-0.27 \mathrm{pc})$ are shown in orange dotted ellipses $\left(L_{\mathrm{bol}}=0.4\right.$ $\left.4.7 \times 10^{45} \mathrm{erg} \mathrm{s}^{-1}\right)$. The filled black circle indicates the position of the AGN, corresponding to the kinematic centre of the masers. The radio continuum (grey contours) and the maser emission (filled coloured circles) were extracted from Gallimore et al. (2004) and aligned based on the re-analysis of Gallimore \& Impellizzeri (2019). The radio and IR images are aligned based on the continuum peaks. The green dashed lines indicate the bipolar outflow cone observed in IR polarisation on scales of few hundred mas (Gratadour et al. 2015). The orientation of the polarisation cone is very similar to the large scale ionisation cone found by Das et al. (2006). The grey dashed ellipse indicates the observed $10 \mu \mathrm{m}$ emission size (taken from Raban et al. 2009). The elliptical beam, used for the reconstruction, is shown in the lower left corner.

molecules (Lo 2005). Energy and momentum conservation imply that the induced photon has the same frequency and direction as the stimulating photon (Elitzur 1990, 2002). There is a broad consensus that both the maser emission and the NIR emission are powered by the central AGN (e.g. Neufeld \& Melnick 1991). The $\mathrm{X}$-rays are able to heat the gas above the $400 \mathrm{~K}$ threshold where the water abundance is enhanced. To achieve a state in which atomic and molecular phases can co-exist, it is important that the medium is optically thin to the non-masing far-infrared photons, otherwise the trapping of these photons by the maser molecules would lead to a thermalisation of the population. Ultimately, the maser emission rate is limited by the loss or destruction rate of this accompanying far-infrared radiation (Rank et al. 1971; Neufeld et al. 1994). This constrains the geometry of the maser source because the surface area for cooling should be optimised, favouring an elongated or flat structure (Lo 2005).

The fact that mega-masers are almost exclusively found in Seyfert 2 galaxies (Ramolla et al. 2011) is consistent with the picture that disc maser emission is produced and beamed close to the plane of the obscuring material. In NGC 1068, nuclear $\mathrm{H}_{2} \mathrm{O}$ 
Table 2. Parameters of dust sublimation ring.

\begin{tabular}{ll}
\hline \hline Parameter & Value \\
\hline Radius $(r)$ & $0.24 \pm 0.03 \mathrm{pc}$ \\
Thickness $(\delta r)$ & $<0.035 \mathrm{pc}$ \\
Position angle $(\mathrm{PA})$ & $-50^{\circ} \pm 4^{\circ}$ \\
Inclination $(i)$ & $70^{\circ} \pm 5^{\circ}$ \\
\hline
\end{tabular}

$22 \mathrm{GHz}$ maser emission is detected, spanning a velocity range of $600 \mathrm{~km} \mathrm{~s}^{-1}$ nearly symmetric about the systemic velocity of the galaxy (Greenhill \& Gwinn 1997; Gallimore et al. 2001). The maser spots extend out to $\sim 0.8 \mathrm{pc}$, aligned along a position angle of roughly $-45^{\circ}$. While symmetric in velocity, the "red masers" at positive velocities are a factor $\sim 4-5$ brighter than the "blue masers" (Gallimore et al. 2001). In particular $\mathrm{H}_{2} \mathrm{O}$, $22 \mathrm{GHz}$ emission requires hydrogen densities of $\sim 10^{7}-10^{11} \mathrm{~cm}^{-3}$ and dust temperatures above $\sim 400 \mathrm{~K}$, but not too far in excess of 1000-1500 K (Neufeld 2000). The pump rate of different water emission lines $(22,183,321$ and $325 \mathrm{GHz})$ depends sensitively on the local density and temperature (Neufeld 2000). The detection of these higher transitions in the future (e.g. with ALMA) can therefore provide valuable information on the conditions close to the sublimation region.

Unfortunately the absolute astrometric accuracy of the infrared emission (discussed in Sect. 5.1) is not sufficient to align the radio and the infrared emission on the scales of interest for this paper, although the radio continuum and the maser emission can be aligned to $\sim 1.3$ mas accuracy (Gallimore et al. 2004). Given the lack of precise positioning information, one option for the relative alignment would be, given their striking similarities, to match the bright side of the ring-like structure in the $K$-band continuum with the high density of masers spots. However, this does not take into account the implied location of the central black hole. If one does so, a more natural choice is to align the centre of the ring with the kinematic centre of the masers (Gallimore \& Impellizzeri 2019). The overall morphological match between the maser spots, the radio-continuum, and the south-western ridge (side facing the observer) of the $K$-band continuum is remarkable (see Fig. 4). This suggests that the maser and the $K$-band emission originate from the same region, the dust sublimation ring. The fact that maser emission is only detected on the side facing the observer is consistent with the directional beaming of the maser emission (masing clouds are located between the central source and observer). We only find infrared counterparts of the systemic masers and red masers. The weaker blue masers show no infrared counterpart.

\section{Infrared spectral energy distribution}

Our $K$-band image of NGC 1068 shows the central emitting region at an unprecedented spatial resolution. This allows us to estimate the brightness temperature based on the observed NIR and MIR fluxes. The GRAVITY fringe-tracker samples the $K$-band with five spectral elements between 1.95 and $2.35 \mu \mathrm{m}$. The observed flux of NGC 1068 shows a very red colour across the $K$-band. Based on the observed slope we can constrain the effective temperature and the extinction. Figure 5 shows the observed $K$-band flux. We also included the published MIR $(8-13 \mu \mathrm{m})$ SED for the central component (taken from Raban et al. 2009; López-Gonzaga et al. 2014).

In the following subsections, we discuss several models that might explain the observed $K$-band emission. We first provide a short description of the key points of each model, where it can succeed, and where it fails. We focus on whether each of the models can explain the ring-like structure seen in the interferometric data, whether they match the slope of the NIR continuum, and whether they are consistent with other observational constraints (e.g. extinction, luminosity and covering factor). We conclude on which model we favour in Sect. 5, where we provide a heuristic view of the central regions.

Model 1 is a geometrically thick clumpy torus composed of dusty clouds. This class of models is inconsistent with several aspects of the GRAVITY data. Such models, when viewed as close to edge-on as the orientation in NGC 1068 requires, cannot reproduce a ring-like structure. In addition the NIR continuum slope is rather shallower than that observed.

Model 2 enables us to explain both the observed $K$-band and MIR emission with a single, rather cool dust structure of $T_{\text {eff }} \approx$ $720 \mathrm{~K}$. The required NIR luminosity is rather low, and hence so is the implied covering factor, which is naturally the case for a co-planar thin disc. The major difficulty is that this also implies too little foreground extinction.

Model 3 assumes hot dust of $T_{\text {eff }} \approx 1500 \mathrm{~K}$ absorbed by significant foreground extinction. This is mainly motivated by the observed emission size, which suggests hot dust close to the sublimation region. This model requires that the bulk of the MIR continuum originates in separate structures (consistent with the model proposed by Vollmer et al. 2018). Its positive aspects are that it can match the observed steep red slope of the NIR continuum, and that the required foreground extinction is consistent with that expected from other observations. The main difficulty is that the high NIR luminosity requires a large covering factor. But this can be resolved if the accretion disc is tilted with respect to the maser disc as hinted at in point (4) of the introduction.

Model 4 is an alternative to Model 3, in which all of the bright NIR continuum observed by GRAVITY originates from the far side, rather than near-side, of the inner disc. Rather than recognising the $K$-band ring as a physical structure as in Model 3, this model assigns the NIR morphology to chance alignment due to clouds along the line of sight. In particular, it requires unrelated patchy obscuration to create an apparent ringlike structure with a position angle, aspect ratio, and size that match the geometry given by the maser disc and dust sublimation radius.

\subsection{Model 1: geometrically thick clumpy torus}

We have already seen in Sect. 3 that the ring-like structure, especially with the near side brighter than the far side, is hard to reconcile with a geometrically thick clumpy torus model that is close to edge-on. Similarly, these models require a careful matching of the vertical density structure to remain consistent with the presence of the thin maser disc at a radius $\lesssim 1 \mathrm{pc}$.

Here we consider the photometric aspect. We compare our infrared SED to the torus models of Stalevski et al. (2012). These models use a two-phase medium of high density clumps and low-density gas filling the space in between. The model assumes standard silicate and graphite ISM-type grains and does not include a pure graphite grain component at small distances. The models have been calculated for a primary source of $10^{11} L_{\odot}\left(L_{\mathrm{bol}}=0.4 \times 10^{45} \mathrm{erg} \mathrm{s}^{-1}\right)$ with inner and outer radii of 0.5 and $15 \mathrm{pc}$, respectively. We scaled the models to the distance of NGC 1068 and to the observed $K$-band flux. In Fig. 5, models with three different inclinations are compared to the observed SED. We find the best matching models to be at inclinations between $60^{\circ}$ and $70^{\circ}$. Models with higher inclinations 


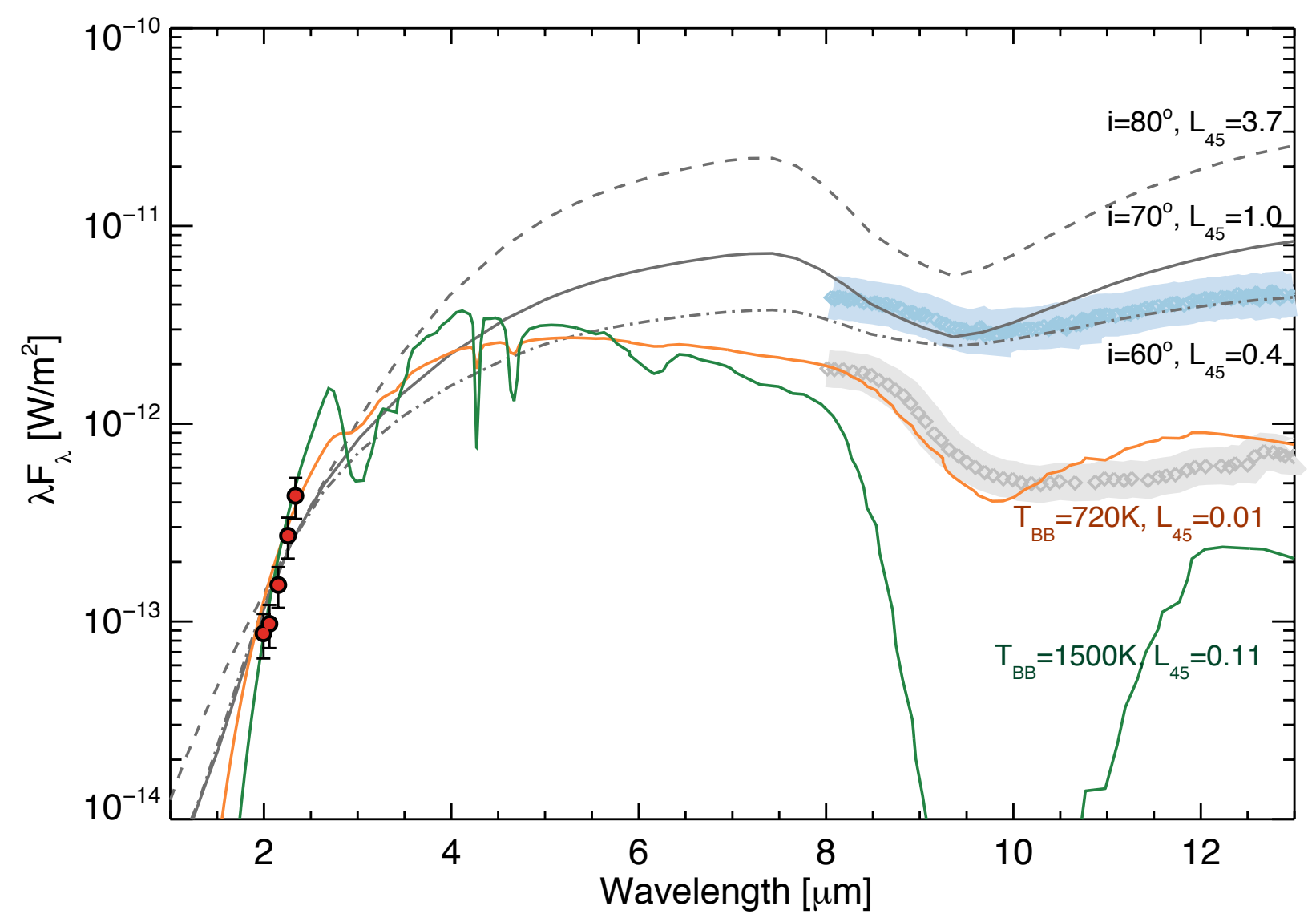

Fig. 5. $K$-band flux density observed with GRAVITY is shown in filled circles. The MIR flux density (incl. uncertainty) taken from Raban et al. (2009) is shown in grey (central 20 mas component only) and in blue (total MIR flux observed with MIDI within few $10 \mathrm{pc}$ ). In black, we show three torus models from Stalevski et al. (2012) with different inclinations of $i=60^{\circ}$ (dash-dotted), $i=70^{\circ}$ (solid) and $i=80^{\circ}$ (dashed). The two single temperature black body models are overplotted. Model 2 in orange ( $T_{\text {eff }}=720 \mathrm{~K}, r=0.24 \mathrm{pc}$ and $\mathrm{d} r=0.045 \mathrm{pc}$.). Model 3 in green $\left(T_{\text {eff }}=1500 \mathrm{~K}, A_{K}=5.5, r=0.24 \mathrm{pc}\right.$ and $\left.\mathrm{d} r=0.026 \mathrm{pc}\right)$. The bolometric luminosities of the different models are indicated in units of $L_{45}=L_{\text {bol }} / 10^{45} \mathrm{erg} \mathrm{s}^{-1}$.

(e.g. $80^{\circ}$ ) tend to over-predict the MIR emission. Models with lower inclinations, on the other hand, under-predict the MIR emission. These scaled models would imply a bolometric luminosity between $L_{\mathrm{bol}}=0.4-1.0 \times 10^{45} \mathrm{erg} \mathrm{s}^{-1}$ for $i=60^{\circ}-70^{\circ}$, in good agreement with earlier estimates of the bolometric luminosity (see Sect. 2.1). However, they do not match the red slope of the NIR continuum (with the caveat that the model is missing a pure graphite-grain component).

This model has difficulties reproducing the ring-like dust structure, accounting for the maser disc, and a NIR continuum slope that is too shallow. We therefore rule out a geometrically thick clumpy torus model for NGC 1068.

\subsection{Model 2: cool dust disc}

MIR interferometry (Jaffe et al. 2004; Raban et al. 2009; LópezGonzaga et al. 2014) was used to model the central component with $600-800 \mathrm{~K}$ dust. In the cool dust model, we try to explain the observed $K$-band and MIR emission with a single source and temperature. The observed SED can be well approximated by a black body with an effective temperature of $T_{\text {eff }}=720 \pm 30 \mathrm{~K}$ seen through a screen of extinction of $A_{K} \approx 0.9 \pm 0.3$ (see Fig. 5). It is somewhat surprising that it is possible to explain the 2-13 $\mu \mathrm{m}$ SED with a single absorbed black body. In particular, the strong silicate absorption dip at $10 \mu \mathrm{m}$ depends sensitively on the amount of dust and the dust composition. The very good agreement of the model and the observed silicate dip would indicate that the dust composition in NGC 1068 is very similar to the dust in our Galactic Centre.

The minimum emission surface would be at least $\sim 0.068 \mathrm{pc}^{2}$, which would correspond to a ring with $r=0.24 \mathrm{pc}$ and a thickness of $\delta r=0.045 \mathrm{pc}(0.65 \mathrm{mas})$. Such a large thickness would be partially resolved and is therefore barely consistent with the data. A single black body ring would produce 1 percent of the estimated bolometric luminosity $\left(L=\sigma A T^{4}=1 \times 10^{43} \mathrm{erg} \mathrm{s}^{-1}\right)$. This covering factor is consistent with a thin dusty disc having $h / r<0.14$, that is co-planar with the accretion disc even under the assumption of anisotropic emission.

The cool dust model would allow us to explain the near-tomid infrared SED with a surprisingly simple model. However it predicts too little extinction, which is inconsistent with the $\operatorname{Br} \alpha$ constraint from ISO $\left(A_{\mathrm{Br} \alpha}>2.4\right.$, Lutz et al. 2000) as well as the millimetre constraints on the column density (Tacconi et al. 1994; Sternberg et al. 1994; García-Burillo et al. 2017; Imanishi et al. 2018). The low extinction value results in a remarkably low bolometric luminosity of the dust. Moreover, it is hard to explain a lack of a dust emission component with a much higher temperature given the distance of the central ring from the very luminous nucleus. We therefore disfavour the cool dust and low extinction model. 


\subsection{Model 3: hot dust disc}

The size of the observed structure in Fig. 3 suggests a temperature close to the dust sublimation temperature of $T_{\text {eff }}=1500 \mathrm{~K}-$ $1800 \mathrm{~K}$. In order to reproduce the $K$-band slope, we therefore assumed a black body of $T=1500 \mathrm{~K}$, which is highly absorbed (by foreground dust) with a mean $K$-band extinction of $A_{K}=$ $5.5 \pm 1.7$. Figure 5 shows the observed fluxes and the proposed model. The corresponding optical extinction is $A_{V} \approx 90 \pm 30$. This is fully consistent with the extinction to the BLR derived from limits on the $\operatorname{Br} \alpha$ broad line emission by Lutz et al. (2000), as well as other estimates of the column density towards the nucleus noted in Sect. 2.2.

In order to explain the observed $K$-band flux with the hot dust model, the emission surface needs to be at least $\sim 0.042 \mathrm{pc}^{2}$. If the emission is not a black body but a grey body then the emission region can be correspondingly larger. The minimum required surface is equivalent to a ring with $r=0.24 \mathrm{pc}$ and a width of $\delta r=0.026 \mathrm{pc}(0.38$ mas $)$. This means that the observed ring-like structure is sufficient to explain the observed $K$-band flux. The width of the ring would be unresolved, which is also consistent with the observations.

The hot dust model fails to produce the observed MIR emission. This is, in principle, not a problem, but it means that the bulk of the $8-13 \mu \mathrm{m}$ emission must originate from significantly cooler dust at larger radii, which is directly exposed to the central continuum and could be related to the dusty outflow (Cameron et al. 1993; Bock et al. 2000; Vollmer et al. 2018).

The model also requires about 10 percent of the estimated AGN bolometric luminosity to be reprocessed by dust and reemitted at NIR wavelengths $\left(L=\sigma A T^{4}=1.1 \times 10^{44} \mathrm{erg} \mathrm{s}^{-1}\right)$. This would be inconsistent with a system in which the accretion disc and thin dusty maser disc are co-planar due to the small covering factor. However, there are clear indications that these structures are misaligned: the relative position angle of the radio jet and maser disc (see Fig. 8 of Gallimore et al. 2004 for an illustration), as well as the inclination of the maser disc with respect to the orientation of the outflowing clouds (Kishimoto 1999). Adopting anisotropic emission from the accretion disc as described by Netzer (2015) $L \propto \cos (\theta)[1+b \cos (\theta)]$ with $b \simeq 2$, we find that about $10 \%$ of the AGN luminosity is intersected by a surrounding disc with $h / r<0.14$ (see Sect. 3.2) when the accretion disc is tilted by $40^{\circ}$. This tilt is consistent with position angle and inclination discrepancies above. A weaker anisotropy, for example $L \propto \cos (\theta)$, requires a somewhat smaller tilt. This means that the "covering factor" issue, which is often taken to imply a geometrically thick structure can, for the NIR continuum, be entirely accounted for by a warped inner disc at scales $\lesssim 0.1 \mathrm{pc}$.

In the scenario of a thin disc screened by foreground extinction, the visibility of the "central engine" (accretion disc and BLR) inside the sublimation hole is an obvious question. The $K$-band contribution of the accretion disc can be estimated based on the bolometric luminosity $L_{\text {bol }} \approx 1 \times 10^{45} \mathrm{erg} \mathrm{s}^{-1}$, the distance $14.4 \mathrm{Mpc}$, a $\lambda 5100 \AA$ bolometric correction factor $\sim 9$ (Netzer 2015) and a SED slope of the accretion disc of $F_{v} \propto v^{\alpha}$ with $\alpha=-0.44$ (Vanden Berk et al. 2001). Also considering the $A_{K} \sim 5.5$ foreground extinction, we infer a $K$-band accretion disc flux of $\sim 9 \mathrm{mJy}$. The empirical slope of Vanden Berk et al. (2001) is significantly redder than the canonical Shakura \& Sunyaev (1973) slope of $\alpha=0.33$. The $K$-band accretion disc emission for the canonical slope would be much weaker, and so the inferred contribution is probably a conservative upper limit. Other evidence for yet bluer slopes as found by
Kishimoto et al. (2007, 2008) would further enhance that argument. The estimated accretion disc contribution of $\sim 9 \mathrm{mJy}$ corresponds to $6 \%$ or less of the flux in the reconstructed image (Fig. 4). This means that a point-like accretion disc could easily be hidden in the image including the centre of the dust ring.

Both aspects of (1) accretion disc and (2) BLR visibility are consistent with the hot dust scenario. However, in both cases expected signatures are not far from the observational results/limits.

\subsection{Model 4: optically thick hot dusty disc}

Model 3 above places the AGN at the centre of the $K$-band hot dust ring that has the expected position angle and aspect ratio for the inner geometry of NGC 1068 and a size comparable to the dust sublimation radius, and identifies this position with the maser kinematic centre. It implies the NIR continuum comes from both sides of the central disc, with the brightest on the near side while the far side is fainter. This implicitly requires that the re-radiating clouds are optically thin to the hot dust emission. Applying a standard gas-to-dust ratio as described in Sect. 2.2 and the extinction law from Sect. 2.3 mean that $\tau_{2.2 \mu \mathrm{m}}=1$ occurs at a column density of $4 \times 10^{22} \mathrm{~cm}^{-2}$. This is the maximum column density allowed for a typical cloud in the dust disc for those models. In order for the far side of the disc to be fainter, there must be some additional obscuration to that line of sight. The modest factor 4 difference in intensity is plausibly equivalent to 1-2 such clouds. Confirming this alignment requires precise astrometry, which is addressed in Sect. 5.1.

An alternative alignment would be implied if the column density through individual clouds were higher, $N_{\mathrm{H}}=10^{23} \mathrm{~cm}^{-2}$ or more, as inferred from physical considerations of cloud stability in the vicinity of an AGN (Krolik \& Begelman 1988; Vollmer et al. 2004; Hönig \& Beckert 2007), so that they are optically thick at NIR wavelengths. In this scenario, the NIR radiation is anisotropic and primarily emerges from the hot, AGN-facing side of each cloud. As a consequence, a ring consisting of such clouds would be brighter on the far side than the near side. We would be exposed to the directly heated surface of clouds on the far side, while on the near side we would mostly observe the clouds' cool back sides. The hot dust disc is still co-incident with the maser dust, but these phenomena trace different phases of the dusty gas within the disc, so that the alignment shown in Fig. 4 is lost. Models for NGC 1068 constructed in this way could be similar to the disc-wind scenario proposed by Hönig \& Kishimoto (2017) for NGC 3783, which, in contrast to conventional geometrically thick torus models, indicate that the bulk of the MIR continuum arises in a wind. Such a model would posit that the NIR continuum comes from the $\tau_{2.2 \mu \mathrm{m}}=1$ surface of the hot disc that is still geometrically thin, although offset from the equatorial plane according to $h / r$, which could be in the range 0.1-0.2; and that at longer wavelengths pertinent to the masers, the disc is optically thin. In this model, the observed NIR continuum would still extend inwards to the dust sublimation radius, but the brightest spot observed in the $K$-band by GRAVITY would coincide with the far side of the disc. The ring morphology with radius matching the expected dust sublimation radius, the similarity between the maser disc and the $K$-band emission, as well as the alignment of the maser and radio continuum with the $K$-band would all be chance associations. The right panel of Fig. 6 shows the alignment for the optically thick hot dust disc model. The alignment of the GRAVITY data to the disc midplane has been implied by radiative transfer simulations using 

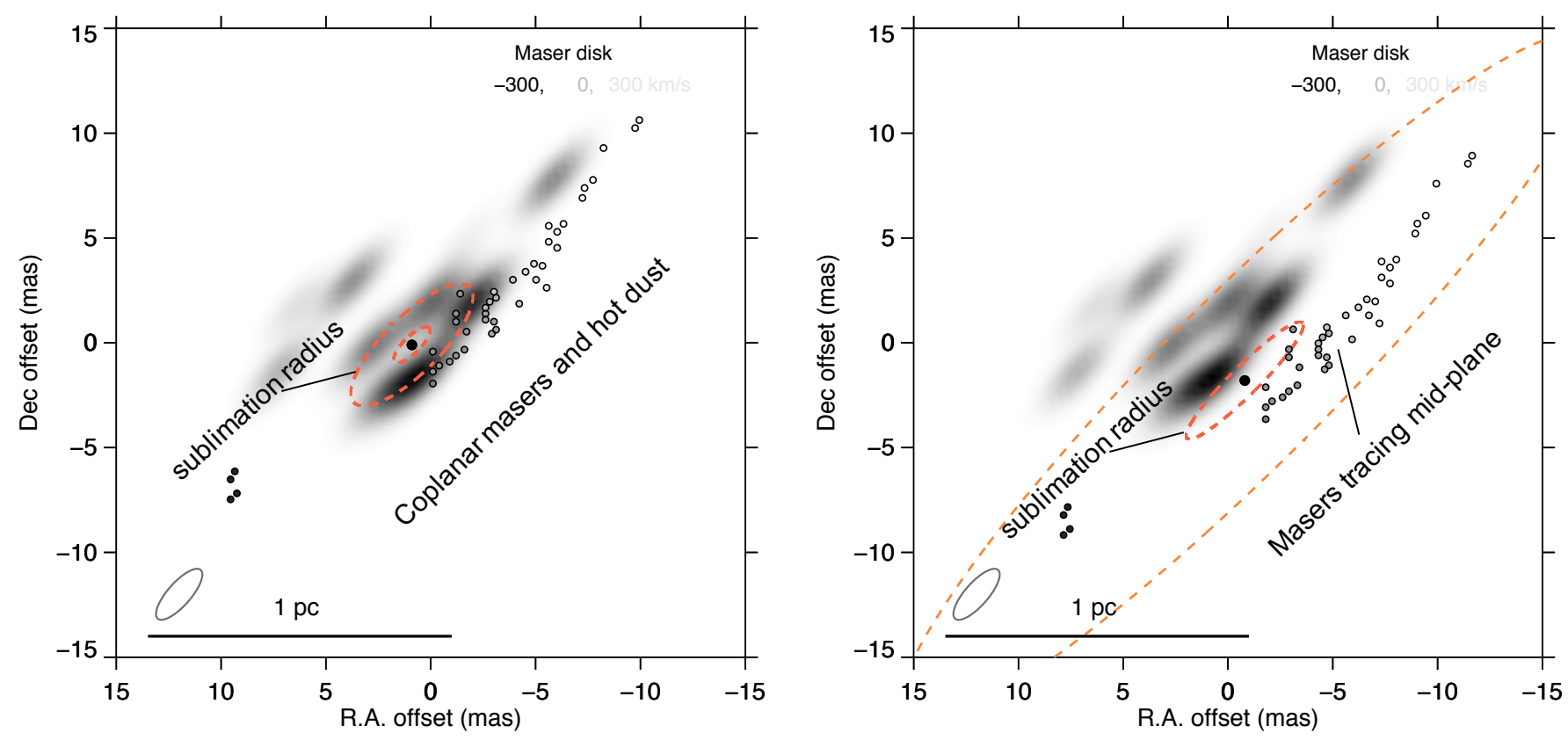

Fig. 6. Comparison of the alignment of the hot dusty disc models. The models themselves are not shown, only the implied interpretation of the observed continuum features. Left: Model 3 is the favoured interpretation, in which the ring-like structure in the $K$-band continuum arises from (partially) optically thin clouds (same as in Fig.4). In this case, it traces a disc that has a similar position angle and aspect ratio to the inner geometry of NGC 1068, is co-planar with the maser emission and traces the dust sublimation ring. Right: Model 4 comprises optically thick clouds, and the alignment of the mid-plane (indicated by the large dashed ring) to the NIR continuum observed with GRAVITY is given by radiative transfer simulations using the CAT3D-WIND model (Hönig \& Kishimoto 2017). In Model 4, the near side of the mid-plane as traced by the masers, but is completely obscured in the $K$-band. The $K$-band emission originates from the $\tau=1$ surface of the hot disc, that is still geometrically thin. But all the structure in the image is assigned to chance effects of patchy obscuration along the line of sight.

the CAT3D-WIND model (Hönig \& Kishimoto 2017) with an assumed inclination of $i=80 \mathrm{deg}$, a scale height of the disc of $h / r=0.1$, and the optical depth of an individual cloud of $\tau_{V}=50$.

For this model, the resulting structures originate with the random distribution of clouds, and hence are due to variations in obscuration along the line of sight. As such, each instance of this model that is created will look different. This makes the model seem arbitrary because one needs to create many such instances in order to pick one that approximates the characteristics of the observed continuum distribution.

\section{Discussion}

\subsection{Astrometry}

The required accuracy to make a decisive statement on the astrometry of the $K$-band continuum with respect to the masers or radio continuum is on the order $\leq 2-3$ mas. Without reference features, which can be identified simultaneously in the $K$-band and in the radio, absolute astrometry is required to relate the radio and infrared measurements. The radio position is sufficiently well known with an uncertainty $\sigma<1.5$ mas (Gallimore et al. 2004). Unfortunately the astrometric uncertainty of the infrared data is a factor 10 worse $(\sigma \sim 20$ mas). Future interferometric observations should be able to exploit nearby stars from the Gaia catalogue as astrometric references. Then, by constructing a local baseline solution using these reference stars and by logging the optical path difference between the stars and NGC 1068, a sufficiently accurate astrometric solution might be achieved.

Without such an astrometric reference yet available, we favour Model 3 which provides a natural explanation for sev- eral aspects of the observed ring-like structure:(i) the match of its position angle and aspect ratio to those of the maser disc; (ii) the match of its radius to the dust sublimation region around the AGN; and (iii) the alignment between its near-side and the masers, when the centre of the ring is aligned to the black hole location derived from the maser kinematics (see Sect. 3.3). The hot dust disc, with clouds that are optically thin in the NIR, is consistent with observations and provides a rationale for the similarity of the masers, radio continuum and the hot dust $K$-band emission. Based on that model we propose a simple disc-like geometry, which is obscured by foreground (within a few parsec) molecular gas.

\subsection{A heuristic model for the central region of NGC 1068}

With a wealth of observations across the electromagnetic spectrum at hand, we try to construct a simple heuristic picture of the central few parsecs of NGC 1068 (see Fig. 7). The $K$-band emission traces the hot dust sublimation radius of a thin disc, seen at an inclination of $70^{\circ}$. The inner masers are cospatial with the $K$-band emission. The masers extend out to radii of $\sim 1 \mathrm{pc}$ (Gallimore et al. 2001), similar to the size of the warm dust structure $(\sim 600 \mathrm{~K})$ seen at MIR wavelengths (Jaffe et al. 2004; Raban et al. 2009; López-Gonzaga et al. 2016). This suggests that the warm dust originates from the outer region of the same thin disc. Beyond $\sim 1 \mathrm{pc}$ distance no masers have been observed, probably because the conditions disfavour masing (e.g. too low dust temperature, $<400 \mathrm{~K}$ ). In contrast to the thin disc at scales of $<1$ pc, ALMA observations by Imanishi et al. (2018) reveal a significant amount of molecular gas, which is rotating with $v_{\mathrm{c}} \approx 40-50 \mathrm{~km} \mathrm{~s}^{-1}$ (notably in the opposite sense as the maser disc) at 5-10 pc distance. The comparably large velocity 


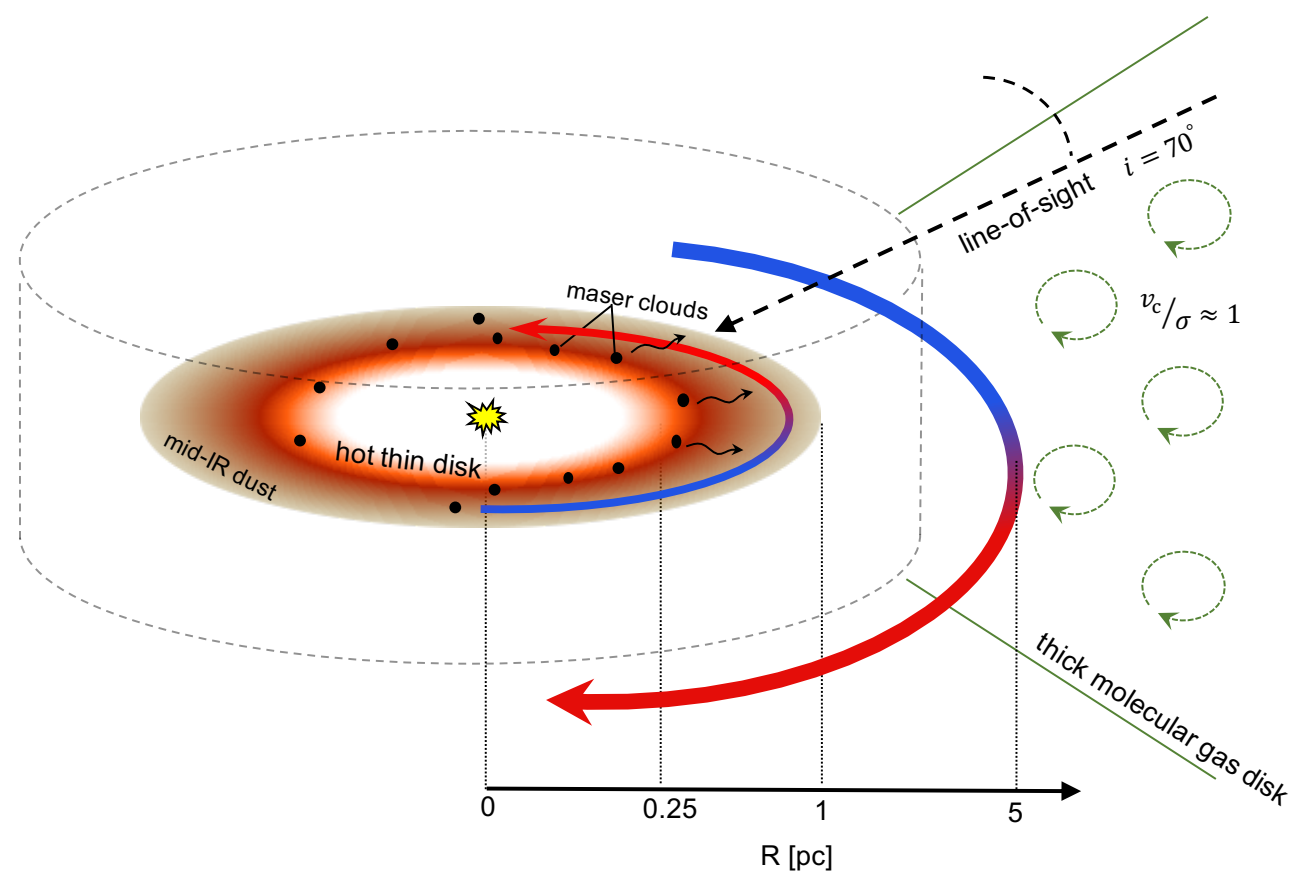

Fig. 7. Sketch of the observed central structures. The $K$-band emission traces the inner rim of a thin disc of hot gas and dust, at or close to the dust sublimation radius $r \approx 0.24 \mathrm{pc}$. The inner water masers are cospatial with the hot $K$-band dust. The masers stretch out to $r \approx 1$ pc (Gallimore et al. 2001). MIR observations show warm dust on similar scales as the outer masers (e.g. Raban et al. 2009), likely originating from the disc periphery. ALMA observations of $\mathrm{HCN}$ and $\mathrm{HCO}^{+}$show a turbulent structure, which rotates in the opposite direction as the maser disc (Imanishi et al. 2018). The $v_{\mathrm{c}} / \sigma$ of the molecular gas structure argues for a thick disc, which contains enough gas mass to reach column densities of $N_{\mathrm{H}} \approx 10^{23} \mathrm{~cm}^{-2}$ that screen the central region by $A_{V} \approx 90$ $\left(A_{K} \approx 5.5\right)$ from the observer. dispersion of $\sigma \approx 30-40 \mathrm{~km} \mathrm{~s}^{-1}$ and the correspondingly high $\sigma / v_{\mathrm{c}} \simeq 1$ suggests a thick disc with a large scale height of $h_{\mathrm{z}} / R \simeq 1$. The disc contains enough gas mass to reach column densities of $N_{\mathrm{H}} \approx 10^{23 . .24} \mathrm{~cm}^{-2}$ that screen the central region by $A_{V} \approx 90\left(A_{K} \approx 5.5 \pm 1.7\right)$. The central few parsecs are fed by two gas streamers (Müller Sánchez et al. 2009), which come very close to the nucleus. The northern and southern streamers reach pericentre distances of $\approx 5 \mathrm{pc}$ and $\approx 1 \mathrm{pc}$ repectively. Their opposite sense of rotation could contribute to the turbulence observed in the molecular disc.

\section{Conclusion}

We present new $0.2 \mathrm{pc}$ resolution observations from the GRAVITY interferometer on the VLT, which spatially resolve the hot dust continuum in the central parsec of NGC 1068. The following conclusions assume a distance of $14.4 \mathrm{Mpc}$. We find that:

- The dust structure is dominated by a thin, ring-like structure with a radius of $r=0.24 \pm 0.03 \mathrm{pc}$, a scale height $h / r<0.14$, an inclination $i=70^{\circ} \pm 5^{\circ}$ and a position angle of PA $=$ $-50^{\circ} \pm 4^{\circ}$.

- The spatial scale of the ring agrees with the predicted sublimation distance of graphite dust illuminated by a $L_{\mathrm{bol}} \approx 0.4-$ $4.7 \times 10^{45} \mathrm{erg} \mathrm{s}^{-1}$ AGN (radiating at or in excess of its Eddington limit). We therefore associate this with the dust sublimation region.

- The NIR continuum structures show a striking resemblance to the maser disc. We suggest that both types of emission originate from the same region at $\sim 0.4 \mathrm{pc}$ distance from the central source. The maser disc is co-spatial with the south-western rim of the dust sublimation region, the side closer to the observer (as expected due to the maser beaming).

- Based on the observed ring-like structure and steep NIR continuum slope, we disfavour geometrically thick clumpy torus models (which also do not account for the maser disc).

- The dust structure and photometry are consistent with a simple model of hot dust at $T \sim 1500 \mathrm{~K}$ that is behind $A_{K} \sim 5.5$ $\left(A_{V} \approx 90\right)$ mag of foreground extinction. This amount of screen extinction is expected from an upper limit to the $\operatorname{Br} \alpha$ broad line and could be provided by the dense and turbulent gas distribution observed on scales of $1-10 \mathrm{pc}$. The model implies that the NIR and MIR emitting dust are in spatially separate locations (the maser disc and the outflow respectively).

Acknowledgements. We thank the referees for their careful reading of the manuscript and their suggestions that have helped to improve it. This research has made use of the NASA/IPAC Extragalactic Database (NED), which is operated by the Jet Propulsion Laboratory, California Institute of Technology, under contract with the National Aeronautics and Space Administration. This work has made extensive use of the software QFitsView, which has been developed by Thomas Ott, Max Planck Institute for Extraterrestrial Physics (http: //www.mpe.mpg.de/ ott/QFitsView/). F. E. and O. P. acknowledge support from ERC synergy grant No. 610058 (Black-HoleCam). J. D. was supported by a Sofja Kovalevskaja award from the Alexander von Humboldt foundation and in part by NSF grant AST 1909711. A. A. and P. G. acknowledge funding from Fundação para a Ciência e a Tecnologia through grants UID/FIS/00099/2013 and SFRH/BSAB/142940/2018. P.O.P acknowledges support from the Programme National Hautes Energies (PNHE) of the Centre National de la Recherche Scientifique (CNRS).

\section{References}

Antonucci, R. 1993, ARA\&A, 31, 473

Antonucci, R. R. J. 1982, Nature, 299, 605

Antonucci, R. R. J. 1984, ApJ, 278, 499

Antonucci, R. R. J., \& Miller, J. S. 1985, ApJ, 297, 621

Anugu, N., Amorim, A., Gordo, P., et al. 2018, MNRAS, 476, 459

Asmus, D., Gandhi, P., Hönig, S. F., Smette, A., \& Duschl, W. J. 2015, MNRAS, 454, 766

Barvainis, R. 1987, ApJ, 320, 537

Baskin, A., \& Laor, A. 2018, MNRAS, 474, 1970

Bauer, F. E., Arévalo, P., Walton, D. J., et al. 2015, ApJ, 812, 116

Bland-Hawthorn, J., Gallimore, J. F., Tacconi, L. J., et al. 1997, Ap\&SS, 248, 9

Bock, J. J., Neugebauer, G., Matthews, K., et al. 2000, AJ, 120, 2904

Cameron, M., Storey, J. W. V., Rotaciuc, V., et al. 1993, ApJ, 419, 136

Das, V., Crenshaw, D. M., Kraemer, S. B., \& Deo, R. P. 2006, AJ, 132, 620

Davies, R. I., Burtscher, L., Rosario, D., et al. 2015, ApJ, 806, 127

Elitzur, M. 1990, ApJ, 363, 628

Elitzur, M. 2002, in Cosmic Masers: From Proto-Stars to Black Holes, eds. V.

Migenes, \& M. J. Reid, IAU Symp., 206, 452

Fritz, T. K., Gillessen, S., Dodds-Eden, K., et al. 2011, ApJ, 737, 73

Gallimore, J. F., \& Impellizzeri, C. M. V. 2019, ApJ, submitted 
Gallimore, J. F., Baum, S. A., \& O’Dea, C. P. 1996a, ApJ, 464, 198

Gallimore, J. F., Baum, S. A., O’Dea, C. P., Brinks, E., \& Pedlar, A. 1996b, ApJ, 462,740

Gallimore, J. F., Henkel, C., Baum, S. A., et al. 2001, ApJ, 556, 694

Gallimore, J. F., Baum, S. A., \& O’Dea, C. P. 2004, ApJ, 613, 794

Gallimore, J. F., Elitzur, M., Maiolino, R., et al. 2016, ApJ, 829, L7

García-Burillo, S., Combes, F., Ramos Almeida, C., et al. 2016, ApJ, 823, L12

García-Burillo, S., Viti, S., Combes, F., et al. 2017, A\&A, 608, A56

Gratadour, D., Rouan, D., Grosset, L., Boccaletti, A., \& Clénet, Y. 2015, A\&A, $581, \mathrm{~L} 8$

GRAVITY Collaboration (Abuter, R., et al.) 2017, A\&A, 602, A94

GRAVITY Collaboration (Dexter, J., et al.) 2019, A\&A, submitted, ArXiv e-prints [arXiv:1910.00593]

Greenhill, L. J., \& Gwinn, C. R. 1997, AP\&SS, 248, 261

Greenhill, L. J., Gwinn, C. R., Antonucci, R., \& Barvainis, R. 1996, ApJ, 472 L21

Güver, T., \& Özel, F. 2009, MNRAS, 400, 2050

Hönig, S. F. 2019, ApJ, 884, 171

Hönig, S. F., \& Beckert, T. 2007, MNRAS, 380, 1172

Hönig, S. F., \& Kishimoto, M. 2017, ApJ, 838, L20

Hönig, S. F., Beckert, T., Ohnaka, K., \& Weigelt, G. 2006, A\&A, 452, 459

Hönig, S. F., Beckert, T., Ohnaka, K., \& Weigelt, G. 2007, in The Central Engine of Active Galactic Nuclei, ed. L. C. Ho, ASP Conf. Ser., 373, 487

Hönig, S. F., Prieto, M. A., \& Beckert, T. 2008, A\&A, 485, 33

Huré, J.-M. 2002, A\&A, 395, L21

Imanishi, M., Nakanishi, K., Izumi, T., \& Wada, K. 2018, ApJ, 853, L25

Impellizzeri, C. M. V., Gallimore, J. F., Baum, S. A., et al. 2019, ApJ, 884, L28

Jaffe, W., Meisenheimer, K., Röttgering, H. J. A., et al. 2004, Nature, 429, 47

Kishimoto, M. 1999, ApJ, 518, 676

Kishimoto, M., Hönig, S. F., Beckert, T., \& Weigelt, G. 2007, A\&A, 476, 713

Kishimoto, M., Antonucci, R., Blaes, O., et al. 2008, Nature, 454, 492

Kishimoto, M., Hönig, S. F., Antonucci, R., et al. 2011, A\&A, 527, A121

Kishimoto, M., Hönig, S. F., Antonucci, R., et al. 2013, ApJ, 775, L36

Koshida, S., Minezaki, T., Yoshii, Y., et al. 2014, ApJ, 788, 159

Krolik, J. H., \& Begelman, M. C. 1988, ApJ, 329, 702

Lacour, S., Dembet, R., Abuter, R., et al. 2019, A\&A, 624, A99

Lapeyrere, V., Kervella, P., \& Lacour, S. 2014, in Optical and Infrared Interferometry IV, Proc. SPIE, 9146, 91462D

Lo, K. 2005, Annu. Rev. Astron. Astrophys., 43, 625

Lodato, G., \& Bertin, G. 2003, A\&A, 398, 517

López-Gonzaga, N., Jaffe, W., Burtscher, L., Tristram, K. R. W., \& Meisenheimer, K. 2014, A\&A, 565, A71

López-Gonzaga, N., Burtscher, L., Tristram, K. R. W., Meisenheimer, K., \& Schartmann, M. 2016, A\&A, 591, A47

Lopez-Rodriguez, E., Fuller, L., Alonso-Herrero, A., et al. 2018, ApJ, 859, 99

Lutz, D., Genzel, R., Sturm, E., et al. 2000, ApJ, 530, 733

Marconi, A., Risaliti, G., Gilli, R., et al. 2004, MNRAS, 351, 169

Marinucci, A., Bianchi, S., Matt, G., et al. 2016, MNRAS, 456, L94

Matt, G., Guainazzi, M., Frontera, F., et al. 1997, A\&A, 325, L13

Miller, J. S., \& Antonucci, R. R. J. 1983, ApJ, 271, L7

Miller, J. S., Goodrich, R. W., \& Mathews, W. G. 1991, ApJ, 378, 47

Mor, R., \& Netzer, H. 2012, MNRAS, 420, 526

Mould, J. R., Huchra, J. P., Freedman, W. L., et al. 2000, ApJ, 529, 786

Müller Sánchez, F., Davies, R. I., Genzel, R., et al. 2009, ApJ, 691, 749

Nenkova, M., Sirocky, M. M., Nikutta, R., Ivezić, Ž., \& Elitzur, M. 2008, ApJ, 685,160

Netzer, H. 2009, MNRAS, 399, 1907

Netzer, H. 2015, ARA\&A, 53, 365

Neufeld, D. A. 2000, ApJ, 542, L99

Neufeld, D. A., \& Melnick, G. J. 1991, ApJ, 368, 215

Neufeld, D. A., Maloney, P. R., \& Conger, S. 1994, ApJ, 436, L127

Packham, C., Young, S., Hough, J. H., Axon, D. J., \& Bailey, J. A. 1997, MNRAS, 288, 375

Pfuhl, O., Haug, M., Eisenhauer, F., et al. 2012, in Optical and Infrared Interferometry III, Proc. SPIE, 8445, 84451U

Pfuhl, O., Haug, M., Eisenhauer, F., et al. 2014, in Optical and Infrared Interferometry IV, Proc. SPIE, 9146, 914623

Pier, E. A., \& Krolik, J. H. 1992, ApJ, 401, 99

Planck Collaboration XIII. 2016, A\&A, 594, A13

Planesas, P., Scoville, N., \& Myers, S. T. 1991, ApJ, 369, 364

Predehl, P., \& Schmitt, J. H. M. M. 1995, A\&A, 293, 889

Prieto, M. A., Reunanen, J., Tristram, K. R. W., et al. 2010, MNRAS, 402, 724

Raban, D., Jaffe, W., Röttgering, H., Meisenheimer, K., \& Tristram, K. R. W. 2009, MNRAS, 394, 1325
Ramolla, M., Haas, M., Bennert, V. N., \& Chini, R. 2011, A\&A, 530, A147

Rank, D. M., Townes, C. H., \& Welch, W. J. 1971, Science, 174, 1083

Ricci, C., Trakhtenbrot, B., Koss, M. J., et al. 2017, ApJS, 233, 17

Risaliti, G., Elvis, M., \& Nicastro, F. 2002, ApJ, 571, 234

Rouan, D., Grosset, L., \& Gratadour, D. 2019, A\&A, 625, A100

Schnülle, K., Pott, J. U., Rix, H. W., et al. 2015, A\&A, 578, A57

Shakura, N. I., \& Sunyaev, R. A. 1973, A\&A, 24, 337

Stalevski, M., Fritz, J., Baes, M., Nakos, T., \& Popović, L. Č. 2012, MNRAS, 420, 2756

Sternberg, A., Genzel, R., \& Tacconi, L. 1994, ApJ, 436, L131

Storchi-Bergmann, T., Kinney, A. L., \& Challis, P. 1995, ApJS, 98, 103

Suganuma, M., Yoshii, Y., Kobayashi, Y., et al. 2006, ApJ, 639, 46

Tacconi, L. J., Genzel, R., Blietz, M., et al. 1994, ApJ, 426, L77

Thiébaut, E. 2008, in Optical and Infrared Interferometry, Proc. SPIE, 7013, $70131 \mathrm{I}$

Thiébaut, É., \& Young, J. 2017, J. Opt. Soc. Am. A, 34, 904

Vanden Berk, D. E., Richards, G. T., Bauer, A., et al. 2001, AJ, 122, 549

Vollmer, B., Beckert, T., \& Duschl, W. J. 2004, A\&A, 413, 949

Vollmer, B., Schartmann, M., Burtscher, L., et al. 2018, A\&A, 615, A164

Weigelt, G., Wittkowski, M., Balega, Y. Y., et al. 2004, A\&A, 425, 77

Winter, L. M., Veilleux, S., McKernan, B., \& Kallman, T. R. 2012, ApJ, 745, 107

Wittkowski, M., Kervella, P., Arsenault, R., et al. 2004, A\&A, 418, L39

1 Max Planck Institute for Extraterrestrial Physics(MPE), Giessenbachstr.1, 85748 Garching, Germany e-mail: davies@mpe.mpg.de

2 LESIA, Observatoire de Paris, Université PSL, CNRS, Sorbonne Université, Univ. Paris Diderot, Sorbonne Paris Cité, 5 place Jules Janssen, 92195 Meudon, France

3 I. Institute of Physics, University of Cologne, Zülpicher Straße 77, 50937 Cologne, Germany

4 Departments of Physics and Astronomy, Le Conte Hall, University of California, Berkeley, CA 94720, USA

5 Department of Physics and Astronomy, University of Southampton, Southampton, UK

6 Department of Physics, Kyoto Sangyo University, Kita-ku, Japan

7 Université Côte d'Azur, Observatoire de la Côte d'Azur, CNRS, Laboratoire Lagrange, Nice, France

8 School of Physics and Astronomy, Tel Aviv University, Tel Aviv 69978, Israel

9 Department of Astronomy, The Ohio State University, Columbus, $\mathrm{OH}, \mathrm{USA}$

10 Center for Cosmology and AstroParticle Physics, The Ohio State University, Columbus, OH, USA

11 Space Telescope Science Institute, Baltimore, MD, USA

12 Univ. Grenoble Alpes, CNRS, IPAG, 38000 Grenoble, France

13 School of Physics and Astronomy, Tel Aviv University, Tel Aviv 69978, Israel

14 Center for Computational Astrophysics, Flatiron Institute, 162 th Ave., New York, NY 10010, USA

15 European Southern Observatory, Casilla 19001, Santiago 19, Chile

16 European Southern Observatory, Karl-Schwarzschild-Str. 2, 85748 Garching, Germany

17 Sterrewacht Leiden, Leiden University, Postbus 9513, 2300, RA Leiden, The Netherlands

18 Max Planck Institute for Radio Astronomy, Bonn, Germany

19 Universidade de Lisboa - Faculdade de Ciências, Campo Grande, 1749-016 Lisboa, Portugal

20 Faculdade de Engenharia, Universidade do Porto, rua Dr. Roberto Frias, 4200-465 Porto, Portugal

21 CENTRA - Centro de Astrofísica e Gravitação, IST, Universidade de Lisboa, 1049-001 Lisboa, Portugal

22 Max Planck Institute for Astronomy, Königstuhl 17, 69117 Heidelberg, Germany

23 Instituto de Astrofísica de Canarias (IAC), 38200 La Laguna, Tenerife, Spain 